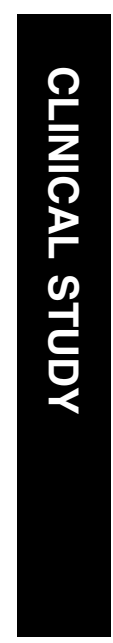

\section{Corneal nerve alterations in acute Acanthamoeba and fungal keratitis: an in vivo confocal microscopy study}

\author{
Abstract \\ Purpose To study sub-basal corneal \\ nerve alterations in patients with acute \\ Acanthamoeba keratitis (AK) and fungal \\ keratitis (FK), using laser in vivo \\ confocal microscopy (IVCM). \\ Methods A retrospective analysis of IVCM \\ (Heidelberg Retina Tomograph 3/Rostock \\ Cornea Module) images of 10 AK corneas and 4 \\ FK corneas was performed, and the results \\ compared with those of $\mathbf{1 0}$ normal and $\mathbf{1 2}$ acute \\ herpetic keratitis (HK) corneas. Sub-basal \\ corneal nerves were analyzed with respect to \\ total number of nerves, main nerve trunks, \\ branching pattern and total length of nerves \\ per image, as well as tortuosity. For each \\ variable, results for three frames were averaged \\ and analyzed using analysis of variance. \\ Results Total corneal nerve length was \\ significantly $(P<0.0001)$ reduced in patients \\ with AK (193.4 $\pm 124.5 \mu \mathrm{m})$ and FK \\ $(268.6 \pm 257.4 \mu \mathrm{m})$ when compared with normal \\ controls $(3811.84 \pm 911.4 \mu \mathrm{m})$. Total nerve \\ counts in patients with AK $(3.9 \pm 1.2)$ and \\ FK $(3.6 \pm 3.2)$ were significantly $(P<0.0001)$ \\ decreased in comparison with normal controls \\ (24.7 \pm 5.5$)$. The number of main nerve trunks \\ and nerve branching was found to be \\ significantly lower in AK and FK corneas, \\ when compared with controls. There was a \\ statistically significant decrease in the above \\ parameters when compared with HK controls. \\ Conclusions The sub-basal corneal nerve \\ plexus is significantly diminished in eyes with \\ AK and FK, as demonstrated by IVCM. These \\ results are more profound than previously \\ reported findings of a diminished nerve \\ plexus in HK. \\ Eye (2012) 26, 126-132; doi:10.1038/eye.2011.270; \\ published online 11 November 2011
}

K Kurbanyan, LM Hoesl, WA Schrems and P Hamrah

Keywords: corneal nerves; Acanthamoeba keratitis; fungal keratitis; in vivo confocal microscopy

\section{Introduction}

The cornea, being one of the most extensively innervated structures in the human body, is supplied by a complex and dense network of sensory, sympathetic, and parasympathetic nerve fibers. ${ }^{1}$ Injury to the nerve fibers can impair corneal health, through poorly understood mechanisms, possibly involving a paucity of neuropeptides that may promote corneal healing. ${ }^{1}$ Reduced corneal nerve function may result in marked reduction in lacrimal gland secretion and tear quality, leading to epitheliopathy and poor epithelial healing. Any disruption of the integrity of corneal innervation, whether from infection or surgery, may lead to transient or chronic neurotrophic keratopathy, which may determine the subsequent prognosis of corneal health.

Neurotrophic keratopathy may result in corneal surface disease or scarring, ${ }^{2}$ leading to subnormal visual acuity and possible blindness. Although herpetic keratitis (HK) is the entity most consistently implicated in neurotrophic keratopathy and loss of the sub-basal nerve plexus, ${ }^{3}$ other infectious disease states may be associated with corneal nerve alterations as well. In Acanthamoeba keratitis (AK) and fungal keratitis (FK), defining corneal nerve involvement on a histopathological level may aid in clarifying the disease processes and in potentially developing novel treatment modalities. 
In vivo confocal microscopy (IVCM) has been used to characterize the in vivo morphology of corneal nerves in great detail. ${ }^{4}$ This technology provides near-histological information with respect to corneal nerves and cellular structures. Recent IVCM studies have demonstrated the feasibility of this technology and have analyzed the sub-basal nerve plexus in normal and diseased corneas. ${ }^{5}$ Prior confocal imaging studies have estimated the presence of 6-8 nerve fiber bundles per image in normal individuals. ${ }^{1}$ Corneal nerve morphology has been demonstrated to be altered in diabetes ${ }^{6}$ and tear deficiency states, such as Sjogren's syndrome. ${ }^{7}$ In addition to the advantage of easily obtainable in vivo characterization of corneal structues, this technology allows longitudinal monitoring of corneal changes.

The purpose of the present study was to describe the confocal microscopic findings in patients with $\mathrm{AK}$ and FK with respect to alterations in corneal nerves: the nerve density, length, tortuosity, and branching pattern have been quantified and compared with normal controls, as well as with corneas with HK.

\section{Materials and methods}

A retrospective review of consecutive patients with AK and FK at the Massachusetts Eye and Ear Infirmary (MEEI) between April 2008 and April 2009 was carried out. For control purposes, 10 corneas of normal eyes and corneas of 12 consecutive patients with acute HK were included in the study. Cases were identified by reviewing microbiology laboratory data for positive culture results, and by searching the confocal microscopy database of the Heidelberg Retinal Tomograph 3/Rostock Cornea Module (HRT3/RCM, Heidelberg Engineering, Dossenheim, Germany) for patients reported to have positive confocal findings consistent with AK (doublewalled cysts) or FK (filamentous hyphae). The diagnosis of herpes simplex keratitis was made according to epithelial dendritic lesions characteristic of epithelial HSV keratitis. All patients had epithelial HK with no stromal involvement. All IVCM studies were performed at the time of acute presentation at the MEEI Ocular Surface Imaging Center, which generally takes $\leq 10 \mathrm{~min}$. Only the first scans at the time of presentation were included. Exclusion criteria for the FK and AK groups were: prior history of infectious keratitis, including herpetic disease, ocular inflammatory disease, ocular trauma, previous eye surgery within 3 months, or diabetes. Exclusion criteria for the herpetic group were: prior history of ocular trauma, ocular surgery within 3 months, contact lens use, or diabetes. Patients receiving local or systemic corticosteroid therapy at the time of the examination were not included. A total of 10 eyes of 10 patients with AK and 4 eyes of 4 patients with
FK were included in the study. Institutional review board (IRB)/ethics committee approval was obtained. This study was complied with the Health Insurance Portability and Accountability Act and adhered to the tenets of the Declaration of Helsinki. Finally, to confirm the appropriate sample size of our study, we performed power calculations, which demonstrated a power of $>95 \%$ for the AK group and $>80 \%$ for the FK group for all statistically significant variables.

\section{Confocal microscopy}

Standard laser IVCM was performed in the affected eyes using the HRT3/RCM (Heidelberg Engineering). One drop of hydroxypropyl methylcellulose $2.5 \%$ (Novartis Ophthalmics, East Hanover, NJ, USA) was placed inside the Tomocap and on the tip of the Tomocap as an optical coupling medium, and the lens was manually advanced until the medium contacted the central surface of the cornea. The confocal microscopy examination was carried out under topical anesthesia with topical $0.5 \%$ proparacaine (Alcon Inc., Fort Worth, TX, USA). In each examination, a full-thickness scan comprising a series of confocal images was obtained through the central cornea by obtaining several sequence and volume scans, as the focal plane was advanced anteroposteriorly. Each image represented a coronal section of approximately $400 \times 400 \mu \mathrm{m}^{2}$ (horizontal $\times$ vertical) and was separated from adjacent images by approximately $2.5 \mu \mathrm{m}$.

\section{Image analysis}

For each patient, three high-quality images of the sub-basal nerve bundle were analyzed per subject. When a corneal ulcer was present with an epithelial defect, the sub-basal area was eroded and no useful scans were available in these patients from the ulcer base,

precluding nerve analysis. We therefore analyzed images of the areas surrounding the ulcer base. The images were selected from the layer immediately at or posterior to the basal epithelial layer and anterior to the Bowman's layer. The criteria to select the images were the best-focused and complete images, with the whole image in the same layer, without motion, without folds, and good contrast. Parameters of corneal nerves in the sub-basal plexus included: (1) the total number of nerves, defined as the number of main nerve trunks and branches present in an image after analyzing the images anterior and posterior to the analyzed image, in order to confirm that these did not branch from other nerves, (2) the nerve branching pattern, defined as the ratio of nerve branches to main nerve trunks in one image, (3) nerve fiber length, defined as the total length of the nerve fibers observed in one image, and (4) the grade of nerve tortuosity, subjectively 
classified into four grades according to the Oliveira-Soto scale: ${ }^{4}$ grade 1 for slight tortuosity, grade 2 for moderate tortuosity with small amplitude curving, grade 3 for more pronounced nerve tortuosity and higher degree of curving, and grade 4 for high amplitude and frequency of curving of the nerve fiber. The nerve analysis was done using Adobe Acrobat software, nerve density was assessed by measuring the total length of the nerve fibers in micrometers per frame $\left(160000 \mu \mathrm{m}^{2}\right)$. The images were viewed in a masked manner without knowledge of patient identity and patient group. Stromal nerves were not analyzed because of inconsistent visualization of the stroma due to infiltrates and edema. Results for three frames were averaged and compared with data from normal controls and eyes with herpetic (simplex and zoster) keratitis. The statistical analysis was carried out using analysis of variance to compare the aforementioned groups.

\section{Results}

Table 1 shows the patient demographics. The patients presented with corneal ulceration centrally/ paracentrally. All patients (10/10) in the AK group and $1 / 4$ patients in the FK group were contact-lens wearers. Cultures of corneal scrapings were positive for the respective diagnoses in 5/10 AK patients and 1/4 FK patients. Diagnoses in all patients were confirmed or established with the demonstration of either doublewalled cystic structures or fungal elements on IVCM studies, which were obtained upon acute presentation.
Imaging of the corneal nerves revealed a marked paucity of nerve structures (see Table 2 for summary). The nerves that were identified were noted to be truncated and lacking the expected branching pattern (Figure 1). The average number of main nerve trunks was decreased in both AK (2.4 \pm 0.7$)$ and FK (2.5 \pm 1.9$)$ groups when compared with normal controls (5.9 \pm 0.7 , $P<0.0001$ ) (Figure 2). Similarly, the total nerve counts in patients with $\mathrm{AK}(3.9 \pm 1.2)$ and FK $(3.6 \pm 3.2)$ were significantly $(P<0.0001)$ reduced in comparison with normal controls ( $24.7 \pm 5.5$; Figure 3$)$. The nerve branching pattern was found to be diminished in AK $(0.6 \pm 0.3)$ and FK $(0.3 \pm 0.4)$ corneas, when compared with normal corneas ( $3.1 \pm 1, P=0.0001$; Figure 4$)$. Total average corneal nerve length per image was found to be $193.4 \mu \mathrm{m}( \pm 124.5)$ in patients with $\mathrm{AK}$ and $268.6 \mu \mathrm{m}$ $( \pm 257.4)$ in patients with FK, which was significantly $(P<0.0001)$ reduced in comparison with normal controls (3811.8 $\pm 911.4 \mu \mathrm{m}$; Figure 5). Tortuosity was significantly increased in the AK $(1.6 \pm 0.6)$ and FK $(2 \pm 1.2)$ groups compared with normal controls $(1.8 \pm 0.7, P=0.0014$; Figure 6). There was a decrease in the number of total nerves $(P<0.0001)$, nerve trunks $(P<0.0001)$, and nerve length $(P<0.0001)$ in AK and FK groups compared with the HK group.

\section{Discussion}

Corneal nerves are known to have an integral role in the recovery following an infectious insult to the cornea, and although the exact mechanisms remain unknown, there may be modulation of the healing process through

Table 1 Demographic data of patients with Acanthamoeba, fungal, and herpetic keratitis in comparison with normal controls

\begin{tabular}{lcccc}
\hline & Acanthamoeba & Fungal & Normal & Herpetic \\
\hline No. of patients $(n)$ & 10 & 4 & 9 (10 eyes) & 7 (12 eyes) \\
Age (mean \pm SD; years) & $41 \pm 13$ & $50 \pm 16$ & $36 \pm 6$ & $61 \pm 13$ \\
Gender (male/female) & $6 / 4$ & $2 / 2$ & $4 / 5$ & $4 / 3$ \\
Days of infection (mean \pm SD) (days) & $14.3 \pm 11.4$ & $15.2 \pm 16.5$ & - & $9.3 \pm 4.5$ \\
\hline
\end{tabular}

Days of infection denotes the time elapsed since diagnosis of the infection until the in vivo confocal microscopy was performed.

Table 2 Corneal nerve parameters in Acanthamoeba and fungal keratitis in comparison with normal and herpetic keratitis control groups $( \pm S D)$

\begin{tabular}{lcccrc}
\hline & Acanthamoeba & Fungal & Normal & Herpetic & P-values \\
\hline No. of main nerve trunks & $2.4(0.7)^{\mathrm{a}}$ & $2.5(1.9)^{\mathrm{a}}$ & $5.9(0.7)$ & $5.9(1.5)$ & $<0.0001$ \\
Branching ratio & $0.6(0.3)^{\mathrm{a}}$ & $0.3(0.4)^{\mathrm{a}}$ & $3.1(1)$ & $1.8(1.1)$ & 0.001 \\
Total number of nerves & $3.9(1.2)^{\mathrm{a}}$ & $3.6(3.2)^{\mathrm{a}}$ & $24.7(5.5)$ & $16.2(6.9)$ & $<0.0001$ \\
Total length $(\mu \mathrm{m})$ & $193.4(124.5)^{\mathrm{a}}$ & $268.6(257.4)^{\mathrm{a}}$ & $3811.8(911.4)$ & $2341.8(720.9)$ & $<0.0001$ \\
Tortuosity & $1.6(0.6)$ & $2(1.2)$ & $1.8(0.7)$ & $2.31(0.6)$ & 0.0014 \\
\hline
\end{tabular}

Values reported as mean $\pm \mathrm{SD}$.

${ }^{a}$ Statistically significant $(P \leq 0.05)$ compared with controls. Statistical analysis was performed by analysis of variance. 

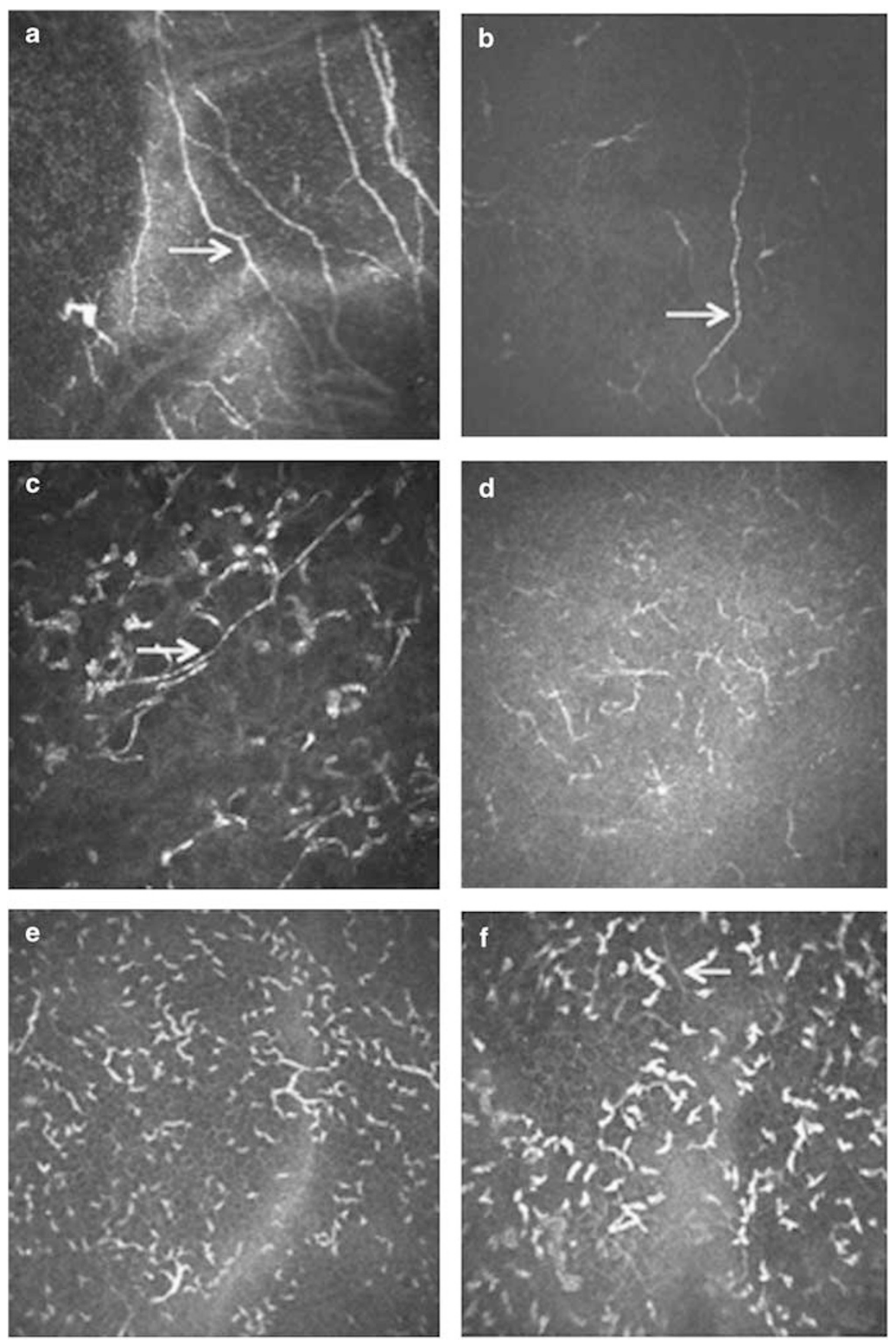

Figure 1 (a) Normal cornea. (b) Herpes simplex keratitis with decrease in nerve length. (c, d) AK with decrease in total nerve count and length. (e, f) FK with decrease in total nerve count and length. Arrows point to nerves. Each image is $400 \times 400 \mu \mathrm{m}$.

release of neuropeptides. ${ }^{1}$ IVCM enables detailed investigation of the sub-basal corneal nerve plexus and can be used to characterize and monitor the extent of damage incurred by infectious keratitis. Although imaging of affected corneas with confocal microscopy is routinely used for diagnostic purposes vis-à-vis identification of characteristic Acanthamoeba cysts $^{8}$ and fungal elements, attention to other cellular and structural details of corneal histology, in particular the corneal nerves, may yield additional information regarding the status and prognosis of corneal health.

The present study demonstrates that central sub-basal corneal nerve density and nerve fiber length are significantly diminished in eyes with acute AK and FK. There is also a marked decrease in nerve branching in these patients. Although it is possible that in the imaging 


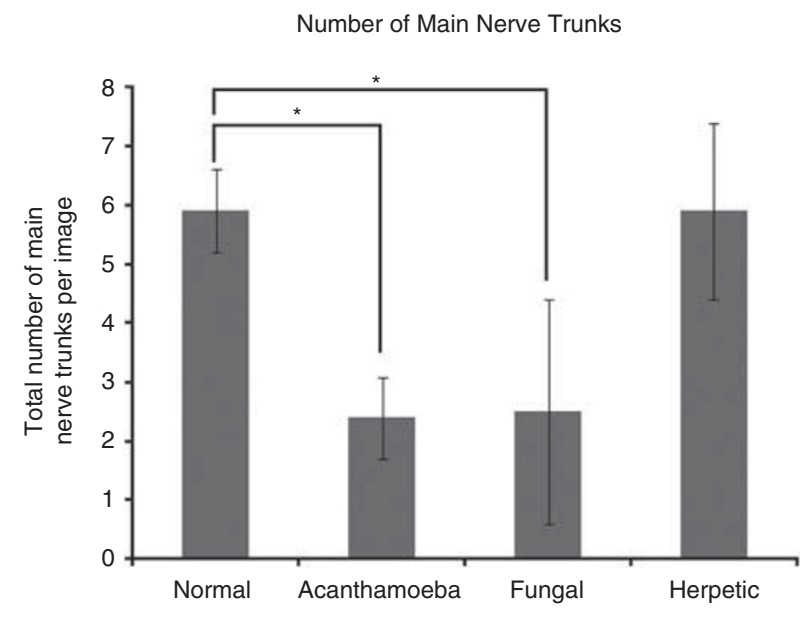

Figure 2 Bar graph showing the number of main nerve trunks in $\mathrm{AK}$ and FK in comparison with normal controls and HK. Error bars represent standard deviation. *Statistically significant $(P \leq 0.05)$ when compared with normal controls.

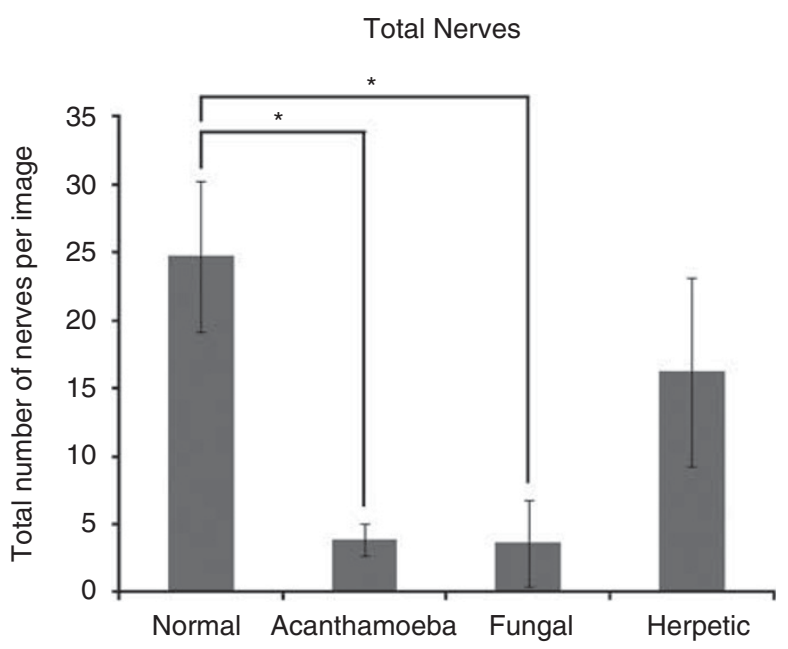

Figure 3 Bar graph showing the number of total nerves in AK and FK in comparison with normal controls and HK. Error bars represent standard deviation. * Statistically significant $(P \leq 0.05)$ when compared with normal controls.

study the nerves may have partially been obscured by the inflammation and fibrosis inherently present in corneas affected by severe keratitis, the profound degree of nerve alterations found in our patient population cannot be attributed to the lack of resolution alone.

At this time, it is unknown whether the observed nerve changes may be caused by severe corneal inflammation alone or may be related to pathogen-induced obliteration of the structures. Although it has been shown that the Acanthamoeba pathogen may directly affect the corneal nerves by possible amoebic infiltration, ${ }^{9}$ further investigation is necessary to establish a definitive cause

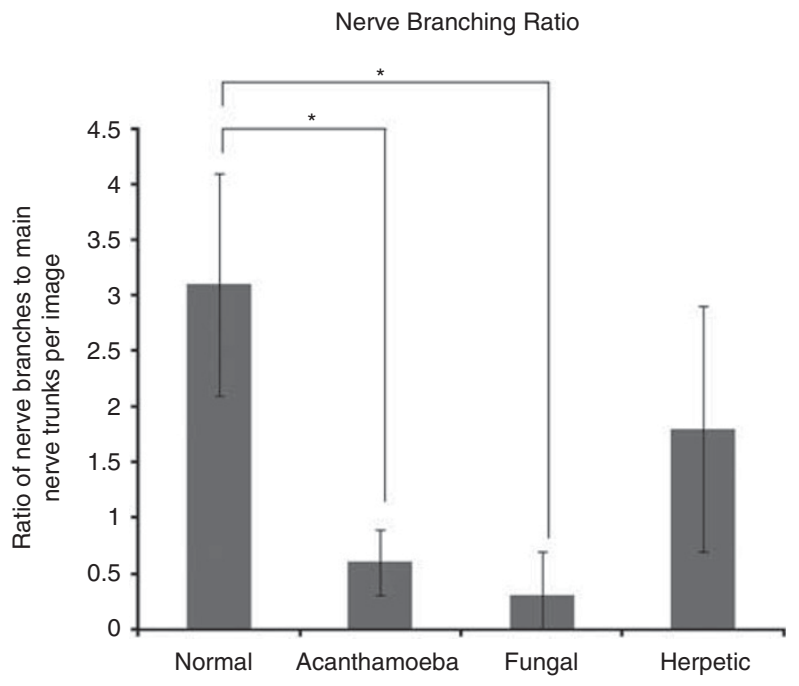

Figure 4 Bar graph showing the nerve branching ratio in AK and FK in comparison with normal controls and HK. Error bar represents standard deviation. *Statistically significant $(P \leq 0.05)$ when compared with normal controls.

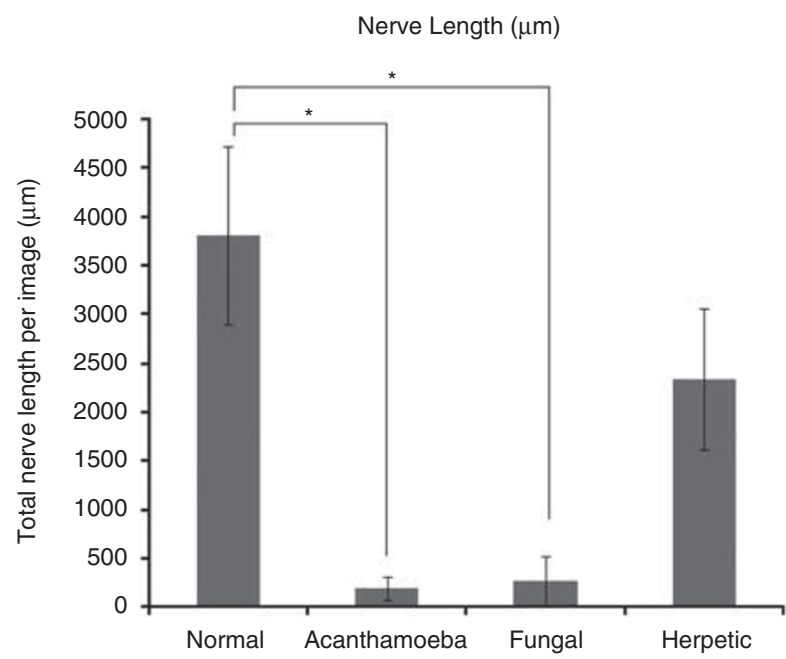

Figure 5 Bar graph showing the total nerve length in AK and FK in comparison with normal controls and HK. Error bars represent standard deviation. * Statistically significant $(P \leq 0.05)$ when compared with normal controls.

for the decrease in nerve density. While the present study focuses on the central cornea, examination of peripheral cornea may further elucidate the status of the corneal nerves in these conditions, which may be especially informative given the known phenomenon of perineuritis observed in Acanthamoeba patients.

Although it has been shown that HK leads to neurotrophic keratopathy with the demonstration of corneal nerve reduction by confocal microscopy, the loss of corneal nerves in AK and FK appears to be more profound compared with changes seen in HK. Unlike the corneal hypoesthesia reported in herpetic infection, 


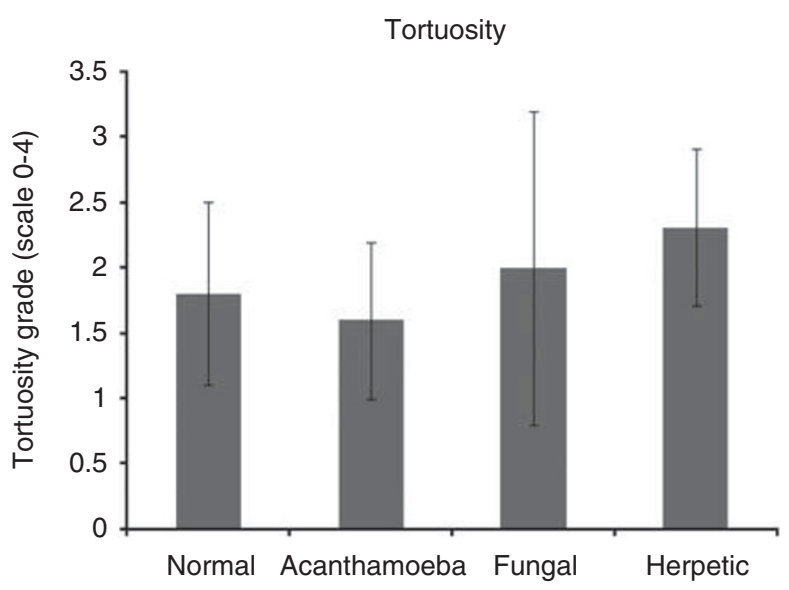

Figure 6 Bar graph showing nerve tortuosity measures in AK and FK in comparison with normal controls and HK. Error bars represent standard deviation.

corneal sensation is not typically measured in patients with AK or FK. On the contrary, patients with AK often present with severe pain. This observation may be explained by possible abnormalities and hypersensitivity in the surviving or regenerating corneal nerves, or the inflammatory pain induced by cytokines (eg, interleukin-1) on nociceptors. There is also a possibility that the pain in these cases may be neurotrophic in origin, with abnormal signaling due to the absence of nerve endings.

Prospective studies of corneal nerves beyond the acute infectious phase may provide information about the long-term outcomes of eyes affected by keratitis. Whether there is regeneration of corneal nerves following the initial phase of the disease is an important consideration in predicting the prognosis of corneal health. Further, previously published studies on sub-basal nerves have demonstrated that sub-basal nerves may be significantly altered or impaired in conditions that affect the integrity of the corneal epithelium, such as bullous keratopathy, ${ }^{10}$ keratoconus, ${ }^{11}$ corneal dystrophies, ${ }^{12}$ and surface neoplasia. ${ }^{13}$ Thus, the diminishment of the sub-basal plexus in AK and FK could at least in part be non-specific owing to epithelial changes or lower visibility of nerves, and thus is not diagnostic. However, the fact that the level of diminishment is severe, argues for an additional role for inflammation in these patients. A recently published prospective IVCM study by our group, demonstrating a strong and significant correlation between the increase in immune cell numbers and the decreased sub-basal corneal nerves in patients with infectious keratitis, confirms the potential role of inflammation. ${ }^{14}$

The Acanthamoeba patients in this study were contact-lens wearers and, although it is known that long-term contact-lens wear may affect the corneal nerves, the observed degree of nerve diminution in the sub-basal plexus would not be expected with currently available soft contact lenses. Studies of corneal innervations in contact-lens wearers have been inconclusive, and, although there are some reports of a decrease in nerve sensation and density, other studies have shown only qualitative differences that were not statistically significant. ${ }^{15}$ Nevertheless, the decrease in corneal density reported in contact-lens wearers is minimal as compared with the significant diminishment observed after AK and FK.

Other limitations of the current study include the small sample size and the retrospective nature of the analysis, which did not allow for measurement of corneal sensation in this population, precluding a clinicopathological correlation. We currently have an ongoing prospective longitudinal study on patients with infectious keratitis, studying changes in corneal sensation and nerve morphology over time in these patients. Another limitation is that the images were interpreted by only one observer. In addition, only the central cornea was imaged with respect to nerve density, and the obtained results cannot be extrapolated to the peripheral cornea, which may show a significantly different distribution and density of corneal nerves.

In conclusion, the study of eyes with acute AK and FK reveals a profound reduction in the central sub-basal corneal nerves. The observation of corneal nerve reduction in these pathological states is of importance in predicting the subsequent healing processes of the cornea. These findings indicate that these corneas are, at least temporarily, neurotrophic and suggest that the practice of measuring corneal sensation to differentiate herpetic from non-herpetic infectious keratitis needs to be reconsidered. Future studies may elucidate the underlying mechanism of corneal nerve abnormalities, which may have important implications in the follow-up and treatment of these corneal conditions.

\section{Summary}

\section{What was known before}

- Decreased nerve density and sensation has been attributed to herpetic keratitis.

What this study adds

- We demonstrate that in patients with Acanthamoeba and fungal keratitis, corneal nerves are severely compromised and diminished, beyond the level of diminishment in patients with herpetic keratitis.

\section{Conflict of interest}

The authors declare no conflict of interest. 


\section{Acknowledgements}

This work was supported by NIH K12-EY016335, NIH K08-EY-020575, the New England Corneal Transplant Research Fund and Falk Medical Research Foundation. The funding organizations had no role in the design or conduct of this research.

\section{References}

1 Müller LJ, Marfurt CF, Kruse F, Tervo TM. Corneal nerves: structure, contents and function. Exp Eye Res 2003; 76: 521-542.

2 Nishida T. Neurotrophic mediators and corneal wound healing. Ocul Surf 2005; 3: 194-202.

3 Hamrah P, Cruzat A, Dastjerdi MH, Zheng L, Shahatit BM, Bayhan HA et al. Corneal sensation and subbasal nerve alterations in patients with herpes simplex keratitis. An in vivo confocal microscopy study. Ophthalmology 2010; 117: 1930-1936.

4 Oliveira-Soto L, Efron N. Morphology of corneal nerves using confocal microscopy. Cornea 2001; 20: 374-384.

5 Patel DV, McGhee CN. In vivo confocal microscopy of human corneal nerves in health, in ocular and systemic disease, and following corneal surgery: a review. Br J Ophthalmol 2009; 93: 853-860.

6 Rosenberg ME, Tervo TM, Müller LJ, Moilanen JA, Vesaluoma MH. In vivo confocal microscopy after herpes keratitis. Cornea 2002; 21: 265-269.
7 Zhang M, Chen J, Luo L, Xiao Q, Sun M, Liu Z. Altered corneal nerves in aqueous tear deficiency viewed by in vivo confocal microscopy. Cornea 2005; 24: 818-824.

8 Matsumoto Y, Dogru M, Sato EA, Katono Y, Uchino Y, Shimmura $S$ et al. The application of in vivo confocal scanning laser microscopy in the management of Acanthamoeba keratitis. Mol Vis 2007; 13: 1319-1326.

9 Pfister DR, Cameron JD, Krachmer JH, Holland EJ. Confocal microscopy findings of Acanthamoeba keratitis. Am J Ophthalmol 1996; 121: 119-128.

10 Al-Aqaba M, Alomar T, Lowe J, Dua HS. Corneal nerve aberrations in bullous keratopathy. Am J Ophthalmol 2011; 151: 840-849.e1.

11 Patel DV, McGhee CN. Mapping the corneal sub-basal nerve plexus in keratoconus by in vivo laser scanning confocal microscopy. Invest Ophthalmol Vis Sci 2006; 47: 1348-1351.

12 Rosenberg ME, Tervo TM, Petroll WM, Vesaluoma MH. In vivo confocal microscopy of patients with corneal recurrent erosion syndrome or epithelial basement membrane dystrophy. Ophthalmology 2000; 107: 565-573.

13 Alomar TS, Nubile M, Lowe J, Dua HS. Corneal intraepithelial neoplasia: in vivo confocal microscopic study with histopathologic correlation. Am J Ophthalmol 2011; 151: 238-247.

14 Cruzat A, Witkin D, Baniasadi N, Zheng L, Jurkunas UV, Ciolino JB et al. Inflammation and the nervous system: the connection in the cornea in patients with infectious keratitis. Invest Ophthalmol Vis Sci 2011; 52: 5136-5143.

15 Oliveira-Soto L, Efron N. Morphology of corneal nerves in soft contact lens wear. A comparative study using confocal microscopy. Ophthalmic Physiol Opt 2003; 23: 163-174. 